\section{Bone physiology}

\section{Hypercalcaemia of malignancy}

\author{
Murray C. Meikle
}

Hypercalcaemia, a complication of malignant diseases, affects up to 40 per cent of patients with certain tumours, the elevation in plasma calcium being caused by tumour products acting on the skeleton to stimulate bone resorption and on the kidney to increase the tubular reabsorption of calcium. Several notable contributions to our understanding of how hypercalcaemia is mediated have been made this year. Probably the most significant development is the recent purification ${ }^{1.2}$ of a parathyroid hormone (PTH)-like peptide from lung and renal carcinomas, whose amino-terminal sequence is very similar to human PTH. And Thompson et al. now provide ${ }^{3}$ direct evidence that this peptide mobilizes bone calcium in vivo. These reports are of particular interest as they support a long-standing hypothesis regarding the pathogenesis of tumourassociated hypercalcaemia proposed by Albright ${ }^{4}$ almost 50 years ago.

The gene for human PTH-related protein (PTH-RP) encodes a 177-aminoacid protein consisting of a 36-amino-acid leader sequence and a 141-amino-acid mature peptide ${ }^{1.2}$. The similarity to PTH, however, is restricted to the aminoterminal region: eight of the first thirteen amino acids of human PTH-RP are identical to human PTH (see figure). Because the amino-terminal region of the human PTH molecule is responsible for its biological activity and binds to the PTH receptor ${ }^{5}$, the similarity of this region seems sufficient to explain the ability of PTH-RP to mimic the effects of PTH. Although PTH-RP is overexpressed by certain tumours, its production is not unique to neoplastic cells. Cultured human keratinocytes also produce PTHRP (ref. 6), which suggests the peptide may be involved in normal skin physiology and explains why tumours of the squamous type are among those most commonly associated with hypercalcaemia. PTH-RP messenger RNA has also been shown ${ }^{7}$ to be expressed in rat mammary tissue, but only during lactation, apparently as a function of the sucking stimulus. This finding is again noteworthy because hypercalcaemia is a common feature of tumours of the breast.

It seems likely that the genes encoding PTH and PTH-RP arose from a common ancestral gene that became duplicated and separated during mammalian evolution. The PTH gene is located on the short arm of chromosome 11 , and the gene encoding PTH-RP has now been mapped' to the short arm of chromosome 12. Chromosomes 11 and 12 are thought to have arisen by tetraploidization from a single ancestral chromosome ${ }^{8}$, and the short arms of both chromosomes carry other functionally related genes: for example, the genes for lactate dehydrogenases $\mathrm{A}$ and $\mathrm{B}$ and the Harvey and Kirsten ras proto-oncogenes (which are on chromosomes 11 and 12, respectively).

In their new work ${ }^{3}$, Thompson et al. mimicked the constant secretion of the peptide by tumours. They continuously administered peptides, either the first 34

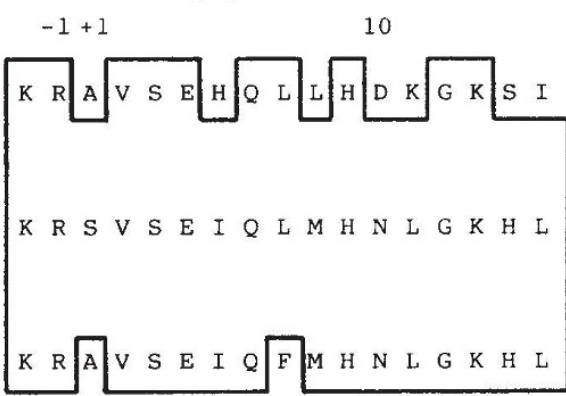

Sequence homologies of the amino-terminal region for human PTH-related peptide (top), human PTH (middle) and bovine PTH (bottom). Eight of the first thirteen amino acids of hPTH-RP are identical to hPTH, which presumably enables the molecule to bind to the PTH receptor: there is little similarity within the remainder of the molecule. (Single-letter amino-acid code.)

residues of bovine PTH, bPTH $(1-34)$, or the equivalent peptide from humans, PTH-RP $(1-34)$, to thyroparathyroidectomized rats fed on a low calcium diet. At an infusion rate of 0.1 nanomoles per hour, both peptides produce hypercalcaemia and nephrocalcinosis and are equally potent in stimulating bone resorption: histomorphometric analysis of tibial long bones reveals a dose-related increase in the number of osteoclast cells. The PTH-RP $(1-34)$ also raises plasma levels of 1,25-dihydroxyvitamin $\mathrm{D}_{3}$ $\left(1,25(\mathrm{OH}), v_{1} \mathrm{D}_{3}\right)$ in the same animal

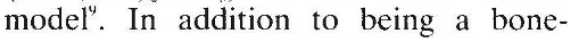
resorbing agent, $1,25(\mathrm{OH})_{2} \mathrm{vitD}_{3}$ also promotes the gastrointestinal absorption of dietary calcium. This finding is likely to be a peculiarity of the animal model, however, because $1,25(\mathrm{OH})$,vitD: levels are usually suppressed in patients with tumour-associated hypercalcaemia, probably by elevated plasma calcium levels or impaired renal function ". An exception appears to be some human lymphotrophic virus type-1 lymphomas associated with osteolytic bone lesions and hypercalcaemia, which do produce $1,25(\mathrm{OH})_{2}$ vitD (ref. 11).

Evidence is also accumulating to link the immunoregulatory cytokines interleukin-1 (IL-1) and lymphotoxin (tumour necrosis factor $-\beta)$ to the hypercalcaemias associated with epithelial carcinomas and haematological malignancies such as myeloma ${ }^{12}$. It has been known for some time that IL-1 can stimulate bone resorption in vitro ${ }^{13}$. Indeed, IL-1 is the most potent agent yet tested in the standard mouse calvarial bone resorption assay and is effective at concentrations as low as 25 picomolar $^{14}$. Experiments conducted on mice by Sabatini el al. ${ }^{15}$ show that continuous infusions of IL-1 markedly increase plasma calcium and osteoclastic bone resorption. Unlike PTH-RP, however, IL-1-mediated hypercalcaemia appears to be exerted solely through its effect on bone: there is no evidence to suggest that IL-1 exerts any effect on renal tubular function.

Although I have focused here on a few specific examples, it is important to stress that many other factors are capable of stimulating bone resorption, at least in vitro, and it is safe to assume that the list will grow. These include tumour necrosis factors $\alpha$ and $\beta$, transforming growth factors $\alpha$ and $\beta$, epidermal growth factor, platelet-derived growth factor, and arachidonic-acid metabolites such as prostaglandins and leukotrienes. The overexpression of any of these factors by neoplastic cells could theoretically be the cause of osteolysis and hypercalcaemia.

Nevertheless, despite their diverse cellular origin and biological function, all these resorptive agents have a common target cell in bone: osteoblasts and not osteoclasts express the receptors for local and systemic resorptive agents. How osteoblasts transmit resorptive signals to osteoclasts remains unclear, and is the subject of intensive investigation because this relationship has important therapeutic implications, not only for the clinical management of tumour osteolysis, but also for other pathological states characterized by bone loss, such as osteoporosis.

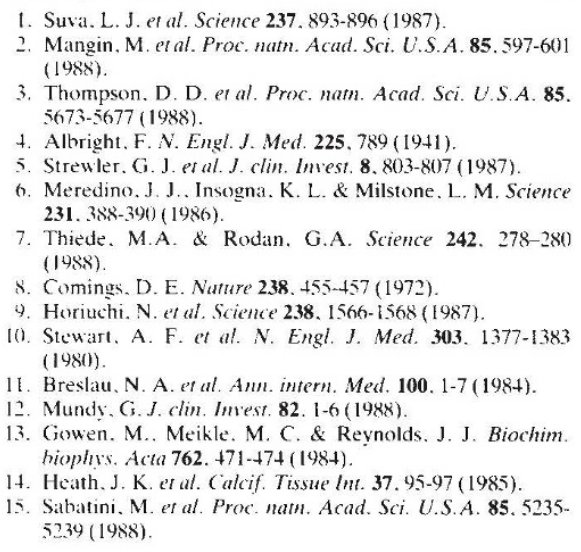

Murrav C. Meikle is at the Strangeways Research Laboratory, Worts Causeway, Cambridge CBI $4 R N, U K$ 